\title{
Dynamics of multiple trapping by a single-beam laser tweezer
}

\author{
Daniel S. Kaputa, Andrey N. Kuzmin, Aliaksandr V. Kachynski, \\ Alexander N. Cartwright, and Paras N. Prasad
}

\begin{abstract}
A multiple-trap single-beam scanning laser tweezer system was developed and characterized. Different stationary and mobile multiple-trap modes were generated for polystyrene beads in a water environment. Trapping efficiency and stability were investigated for several dynamic parameters such as transition time between the sites, waiting time on a single site, number of trapping sites, and IR laser power. Optimal parameters for efficient generation of complex arrays and matrices were determined. We demonstrate an example of a single laser beam multiple-trap application by measuring the trap's stiffness in water for our laser tweezer setup. (C) 2005 Optical Society of America

OCIS codes: $140.7010,120.0120$.
\end{abstract}

\section{Introduction}

For almost two decades laser tweezers have been widely used as precise noncontact optical tools for the investigation of microinteractions within the piconewton force range in many biological and material science experiments..$^{1,2}$ In recent years the development of laser tweezer systems that provide the capability of generating large numbers of trapping sites simultaneously to create two-dimensional (2D) and three-dimensional (3D) arrays of optical traps has greatly extended the capabilities of this tool. These so-called multiple-trap laser tweezers have already been employed for patterning colloidal particles to regular array structures ${ }^{3,4}$ and can be successfully used for many applications, such as photonics, optoelectronics, bioengineering, and colloid physics.

The multiple-trap function of laser tweezers has been demonstrated and reported in a number of papers $^{5-13}$ and was described from the technical point of view in Ref. 12. The multiple-trap action of laser tweezers can be achieved by several methods that can be separated into two groups. The first method entails generating a trap pattern in a specimen plane by

The authors are with The Institute for Lasers, Photonics, and Biophotonics, State University of New York at Buffalo, 458 Natural Sciences Complex, Buffalo, New York 14260-3000. D. S. Kaputa's e-mail address is dskaputa@buffalo.edu.

Received 22 September 2004; revised manuscript received 26 January 2005; accepted 31 January 2005.

0003-6935/05/193963-06\$15.00/0

(C) 2005 Optical Society of America applying intensity space patterning to a stationary, uniform laser beam of light by using an interference, ${ }^{5}$ photomasking, diffractive, ${ }^{6,7}$ phase-contrast, ${ }^{8}$ or holographic ${ }^{9,10}$ technique. The second method involves quickly scanning a tightly focused single laser beam through a number of trap sites. ${ }^{11-13}$

Certainly all the above techniques have some merit, but most publications describe the layouts of the multiple-trap approach, and illustrate multiple trap examples, but do not address the conditions of stability or efficiency during the multiple-trap operation. In this paper we report the development and characterization of a laser scanning multipletrapping system based on the galvano- $X Y$ and piezo- $Z$ scan laser tweezer system described in Ref. 14. In addition to demonstrating a $2 \mathrm{D}$ multiparticle real-time stationary and kinematic manipulation, we also perform an investigation of dynamic trap control parameters. We present several 2D multiple-trap configurations as well as an application of the dualtrap option by measuring trap stiffness.

As mentioned above, the second approach, with a single laser beam control to scan through a number of trap sites, seems to be the simplest, most efficient and convenient way to create a laser tweezer multipletrap operation. In this case, fast repetitive scanning over the pattern permits the production of any $2 \mathrm{D}$ or $3 \mathrm{D}$ structure trap array that one can modify in real time by changing the appropriate trapping parameters by means of a software interface. In comparison to stationary photomasking (amplitude and phase) and computer-controlled spatial light modulation (holographic) methods, with which intensity losses 
could be considerable, this technique does not require a high power laser for generating a trapping-pattern formation. It is also free of diffraction losses and noise, which are inherent in any interference method. The single-laser-beam scanning technique is also more convenient for array construction in that the number of trapping sites as well as each site location can be controlled dynamically.

\section{Laser Tweezers Layout and Software Interface}

Our laser trapping system is based on an optical manipulator (Solar-TII, LM-2), a TEM T0 $_{00}$ cw Nd:YAG laser (Coherent, Compass 1064-2000), and a modified inverted microscope (Nikon, TE-200). A galvano-head (GSI Lumonics, VM500) is used to control the scanning beam and, in addition, to control the beam in any $X-Y$ trajectory (including circular) by the use of a manipulator software interface. An optical trap is formed by a $100 \times$ microscope objective (Nikon-Plane; N.A., $1.3 ; 60 \%$ transmission at $1064 \mathrm{~nm}$ ). The $Z$ position of the trap is controlled by a piezo stage (Piezosystem Jena, MIPOS3SG) with an accuracy within $0.1 \mu \mathrm{m}$. A He-Ne laser, with its beam coincident with the IR, is used as a pilot to visualize the IR trap if necessary. Our optical setup provides the focusing of the IR and red beams at one plane with a spot diameter at the $1 / e^{2}$ level of $<1 \mu \mathrm{m}$. For the experiments we used commercial polystyrene microbeads (Molecular Probes; refractive index, 1.56) of $2.5-\mu \mathrm{m}$ diameter. For our sample, distilled water $(t$ $=25{ }^{\circ} \mathrm{C}$; viscosity, $0.98 \mathrm{cP}$ ) containing the beads was placed between a plain glass slide and a coverslip with a total depth chamber of $100 \mu \mathrm{m}$.

Custom software that interfaces with the GSI Lumonics SC2000 galvano-head scan controller enables us to obtain complex trajectories with the laser tweezer setup. The software was programmed in Visual Basic, and it makes use of the assembler library supplied by GSI Lumonics to send commands serially to the servo controller board. Our software allows us to generate any trajectory for the laser beam and gives us complete control of the transition times and repetition rate between trapping sites. Previously, our software algorithm ${ }^{14}$ generated a program and constantly sent it to the scan controller serially. This technique allowed for the possibility of errors created by interrupts in the serial data transfer caused by the Windows multitasking operating system. The new software approach allows us to generate a program and download the entire object code to the board in one step. The program is stored in the flash memory of the board, and it can run continuously without any reliance on the programming computer. Operating the scan controller as an embedded system allowed the programs to run much faster and also much more reliably without any delayed command execution. The software enables the user to input the desired tweezer parameters, and it computes all the necessary unknowns. The generated program is then sent to the scan controller, where it can be executed.

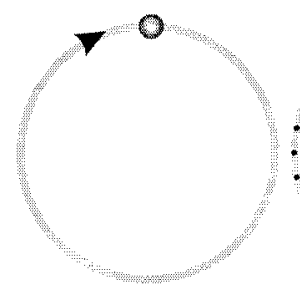

(a)

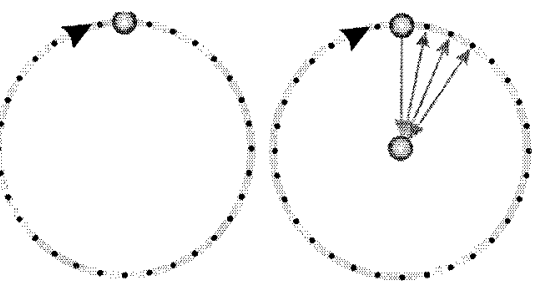

(b)

(c)
Fig. 1. The three distinct laser trajectories used for determining the trapping forces: (a) the continuous mode, (b) the interrupted mode, (c) the complex mode.

\section{Multitrap Dynamics}

For any dynamic real-time controlled multiple-trap option of laser tweezers it is important to know the main dynamic parameters of each trapping site. Choosing the correct parameters can create a stable and predictable trapping behavior of the entire structure during the measurement or for a specific technological application.

To analyze multiple-particle trapping by scanning a single beam of a laser tweezer system, which is inherently a dynamic process, we chose several parameters to adequately describe this process. For a traditional single trap, trapping force and stiffness are the most important characteristics. Usually trapping forces applied to microspheres are calibrated against viscous drag forces exerted by fluid flow. According to this method the trapping forces are calculated by use of the Stokes law on the basis of the escape velocity of the bead from the trap. In the multiple-trap option, evaluation of the trapping forces is much more complicated. One of the ways to study how the dynamics of the scanning beam will influence the stability of multiple-particle trapping is to investigate the trapping stability of a single microsphere under discontinuous motion.

Multiple-trap dynamics were determined with an experimental technique similar to the method described in Ref. 14. A microsphere was rotated in a circular trajectory at a constant angular velocity, and the linear speed of the bead was correspondingly increased as the radius of the trajectory was increased. When the frictional forces of the bead were equal to or greater than the trapping forces, the bead would escape from the trap. We used the radius of the circular trajectory for which the bead escaped to determine the trapping efficiency.

A variation of this technique is that the laser traps the bead on the outer circumference of a circle and then quickly moves to the center of the circle where another particle can be trapped. The laser then returns to the outside of the circle at a point adjacent to the previously trapped outer bead. The outer bead will become trapped again, and in this manner it will move around in a circular trajectory. This is the method that is depicted in Fig. 1(c).

The illumination of the trapped outer bead closely models the interaction of the scanning beam with one trapping site of a multiple-trapping structure. The 
interval of time during which the laser beam travels to the center of the circle models the interaction of the beam with other trapping sites in the multipletrapping structure. In this manner one can determine the dynamics of one trapping site by comparing the escaping radius of this multiple-trap technique to the escaping radius of a uniform-motion circular trajectory where Stokes's law can be used to calculate the trapping forces.

In the above experimental approach we used the tweezers software to create three distinct trajectories, each with its own purpose. All the trajectories traversed by the laser are circular in nature and are composed of line segments that connect 60 to 200 defined points. For a generated trajectory the main parameters are the waiting time per point $t_{w}$, the number of points $n$, the transition time between the points $t_{t}$, and the frequency of the beam rotation $f$. All these parameters are interrelated according to the formula $f=1 /\left[n\left(t_{w}+t_{t}\right)\right]$, and the user can decide which variables are the dependent ones and which are the independent ones.

The first trajectory [Fig. 1(a)], continuous mode, is defined by a slow ( $\sim 1-\mathrm{ms})$ transition time between points and a zero wait time at each point, which results in a continuous motion of the laser and consequently in a continuous motion of the trapped single bead. Under these conditions the trapping force applied to the single bead can be measured by the method described in Ref. 14. This trajectory serves as a reference to which one compares the trajectory that models the multiple-trap phenomena to determine the multiple-trap trapping efficiencies.

The second trajectory [Fig. 1(b)] is similar to that of Fig. 1(a) but with a fast $(\sim 10-\mu \mathrm{s})$ transition time between the points and a long ( $\sim 1-\mathrm{ms})$ waiting time. This results in the laser beam's jumping from point to point, and consequently the trapped single bead moves in a jerking motion from one point to another. When the radius of rotation reaches the escaping radius, which corresponds to the frictional forces equal to or exceeding the trapping forces, the particle will move away from the trapped site, and this escaping radius will characterize the trapping efficiency similarly to the trajectory efficiency. If the distance between neighboring trajectory points is larger then the bead radius, however, the bead will escape from the trap but will remain in the laser path, moving only slowly in a Brownian motion. The laser will return to the point during the next regeneration cycle, and the bead can be trapped again. In this condition the escaping radius cannot characterize the tapping efficiency, and the approach cannot be used. This trajectory [Fig. 1(b)] is referred to as trajectory 2 or the fast transition mode.

In contrast to the previous two, the third trajectory [Fig. 1(c)] is used to truly simulate the multiple-trap phenomenon. This trajectory is the most complex trajectory, in which the laser beam moves from point to point of the circle by passing through the center of the circle. If the linear distance between the neighboring points on the circumference is smaller then the bead

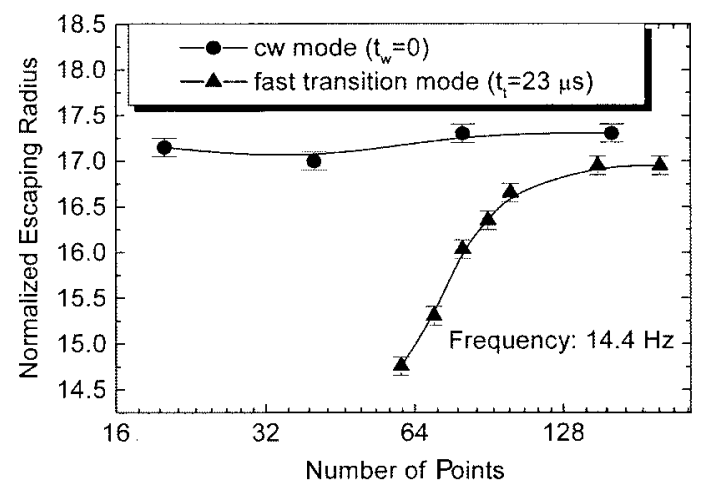

Fig. 2. Escaping radius versus the number of points in the circle trajectory for $\mathrm{cw}$ and fast transition modes of manipulation.

radius and the transition time to and from the middle of the circle is fast enough, the trapped single microsphere will move in a circular trajectory similar to that of the second trajectory.

The illumination of the outer bead jumping between neighboring points on the circumference and the time spent in the center of the circle nicely simulate the interaction of the laser with many particles, i.e., multiple-trap interaction. The experiment described above with only one particle helps to characterize the multiple-trap phenomenon and to allow us to study the optimal combination of dynamic parameters such as waiting and transition time and the number of trapping sites allowed in the trajectory for stable multiple trapping.

For our first experiment, the circular trajectory of the scanning beam (trajectory 2) was used. The trapped microsphere was repetitively rotated along the circle, stopping at each point along the trajectory. The Stokes formula, used in the research described in Ref. 14 for trapping-force calibration, which was derived for a condition of constant particle velocity, could not be directly applied for a spherical particle under discontinuous (nonuniform) motion, which occurs between adjacent trajectory sites. Nevertheless, when the trapped microsphere traveling along this trajectory escaped from the trap, when the radius of the circle became too large (because of high linear velocity and correspondingly large friction), we used the escaping radius parameter ${ }^{14}$ to compare the optical tweezers motions of trajectories 1 and 2 . If the escaping radius for the trajectory 2 mode is equal to or close to that for the trajectory 1 mode under other equal conditions (laser power, temperature, microsphere material and diameter, etc.), the trapping force for the trajectory 2 mode can be approximated in the same way as for the trajectory 1 mode. Even though the Stokes formula cannot be directly applied for a trapping-force estimation for trajectory 2 (nonuniform motion), the escaping radius remains a parameter that characterizes the trapping stability.

We compared two modes of microsphere manipulation described above by investigating the escaping radius and its dependence on the number of points in a circle trajectory (see Fig. 2) for the same frequency 


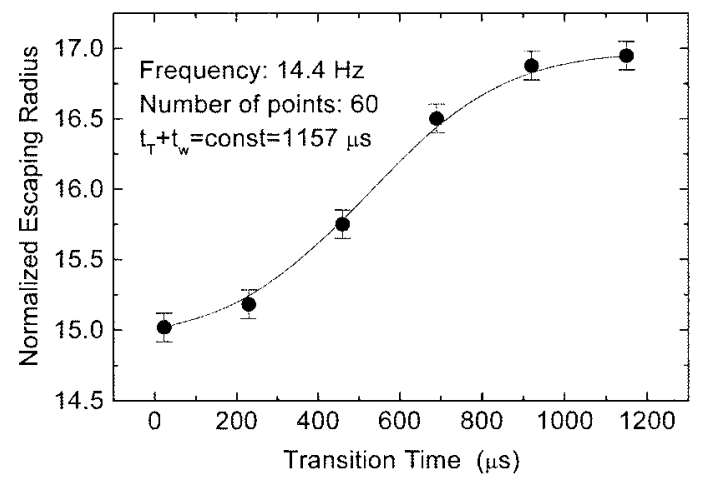

Fig. 3. Dependence of the escaping radius on the transition time between two adjacent neighbor trap points of the circle trajectory.

of trajectory regeneration (angular speed particle rotation) of $f=14.4 \mathrm{~Hz}$ and laser power $P=148 \mathrm{~mW}$. For trajectory $1\left(t_{w}=0\right)$ the escaping radius depends only slightly on the number of points in the trajectory in the range of $20-200$ points (at least at the experimental error level). For trajectory 2, the escaping radius changes weakly in the range of 100-200 points and is close to that for trajectory 1 . The similar escaping radii for experiments with 100 to 200 points is in good correlation with the linear interval between the neighboring points of 0.7-1.25 $\mu \mathrm{m}$ (less then the bead radius). For a number of points fewer than 100 , the escaping radius drops off owing to an increased distance between the neighboring points. The maximum decrease of the escaping radius occurs when the distance between two neighboring points becomes larger than the radius of the microsphere $(>1.25 \mu \mathrm{m})$. A smooth change between trajectory 1 and trajectory 2 modes for $n=60$ is shown in Fig. 3. The frequency of rotation and the laser power for this measurement were kept the same as for the previous experiment. For all the transition times in Fig. 3 the corresponding distance between adjacent points is larger than the radius of the microsphere. Unstable trapping for transition times faster than $900 \mu \mathrm{s}$ takes place most likely as the result of a combination of a large distance between sites compared with the radius of the bead, and a high speed of transition $(>2.4 \mathrm{~mm} / \mathrm{s}$, compared with $1.94 \mathrm{~mm} / \mathrm{s}$ for the $\mathrm{cw}$ mode).

One can draw an important conclusion from the above experiments by comparing the data from trajectory 1 and from trajectory 2 modes of motion. With a high degree of accuracy the data of the trapping efficiency investigation of the continuous motion of trajectory 1 can be used to model the jerking motion of trajectory 2. This comparison can be made under the condition that the distance between the neighboring points is less than the radius of the beads or, for this experiment, that the number of points is greater than 100.

To estimate the stability of trapping for the multiple-trap option we use a complex circle trajectory (trajectory 3 ) of the laser beam. In this multipletrap configuration a time-sharing approach is

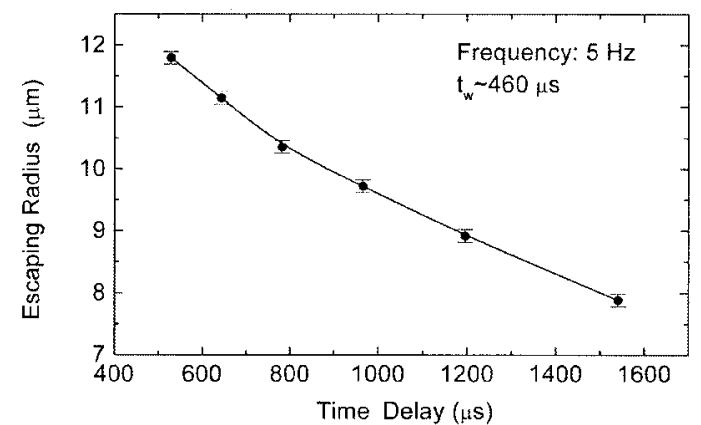

Fig. 4. Dependence of the escaping radius on the transition time from one outer trajectory site to the next in the complex circle trajectory.

employed in which a microbead is illuminated for only a brief time and then the laser moves quickly to the center of the circle, where it could potentially trap another bead. Note that the laser does not have to move to the center of the circle, as this movement is merely used to simulate the time spent at other trapping sites in the multiple-trap configuration. Instead of traveling to the center of the circle the laser could travel to any desired position to achieve the same effect. The movement to the center was chosen purely for the purpose of simplicity. After some amount of time in the center, the beam jumps back to the next point of the outer circular trajectory adjacent to the outer bead. The end result that the user will see is a trapped bead moving about in a circular motion. The dependence of the escaping radius for the outer bead, which was rotated in the outer circle trajectory, on the time of transition (see Fig. 4) is nonlinear and drops faster for a smaller transition time. The average laser power $P_{\text {av }}$, which illuminated the bead during its rotation, is calculated according to the formula $P_{\mathrm{av}}=P\left[t_{w} /\left(t_{w}+t_{t}\right)\right]$, where $P$ is the laser $\mathrm{cw}$ beam power. Figure 5 shows that the calculated average power needed to trap a bead in trajectory 3 is much higher than the power required to trap a bead in trajectory 1 with the same radius. This fact shows that the microsphere's motion in a multiple-trap pattern is not uniform and could not be described directly by the Stokes law in terms of the trapping force but that the escaping radius could be used as a pa-

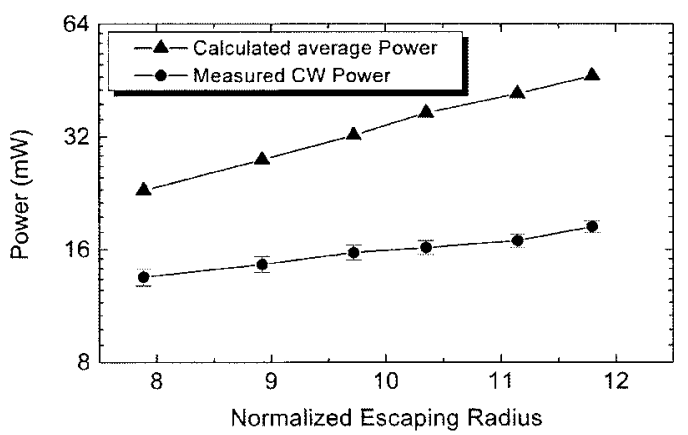

Fig. 5. Calculated average laser power for complex circle trajectory and measured laser power for the cw mode of bead rotation corresponding to the same escaping radius. 

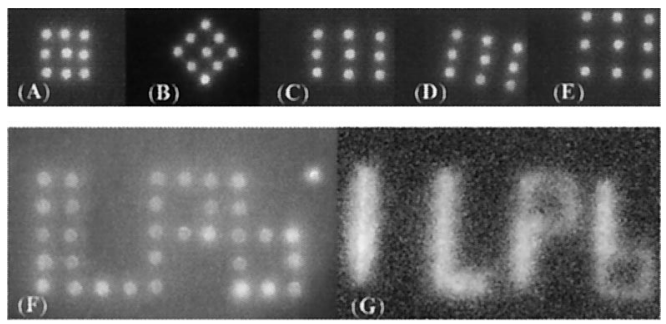

Fig. 6. Microscope images of multitrap option examples: (a)-(e) Real-time dynamic control of the $3 \times 3$ matrix of 2.5 - $\mu \mathrm{m}$ diameter polystyrene beads in water; $t_{t}=69 \mu \mathrm{s}, t_{w}=690, P=196 \mathrm{~mW}$. (f) Stationary trapped 32 beads; $t_{t}=t_{w}=1.15 \mathrm{~ms}$; distance between points, $3.6 \mu \mathrm{m}$. (g) Dynamic image (two-photon fluorescence emission under trapping-beam excitation) of four beads in motion (each bead drawing its own character); integration time of the imaging CCD, $2 \mathrm{~s}$. Dim portions of the letters $\mathrm{p}$ and $\mathrm{b}$ are caused by only a single pass of the scanning laser), $t_{t}=460 \mu \mathrm{s}, t_{w}=1,15 \mathrm{~ms}$; distance between sites, $1.43 \mu \mathrm{m}$.

rameter for the characterization of multiple-trap stability for this nonuniform trap modality. As was previously determined for our setup, to use the escape radius as a means of characterizing the trapping efficiency requires that the number of points used in the experiment be greater then 100 .

To evaluate the maximum number of sites possible with the multiple-trap option of our laser tweezer system we arranged a five-site in-line multiple-trap pattern with a distance of $\sim 7 \mu \mathrm{m}$ between the sites. The stability of the five beads trapped under variations of the time parameters $t_{w}$ and $t_{t}$ was investigated. We found that, for $t_{t}=1.150 \mathrm{~ms}$, the maximum allowable total wait time for stable trapping of the five beads is approximately $t_{w}=161 \mathrm{~ms}$. A simple calculation based on decreasing the waiting time to $t_{w}=t_{t}=1.150 \mathrm{~ms}$ results in the capability of trapping $\sim 350$ beads for these trapping conditions (room temperature, water as a medium, $P$ $=150 \mathrm{~mW}$, and polystyrene $2.5-\mu \mathrm{m}$ beads).

\section{Multiple Trapping for Generating Two-Dimensional Pattern Assemblies}

Several examples of real-time, multiple-trap manipulation of stained polystyrene beads in water are presented in Fig. 6 . The matrix of $3 \times 3$ elements [Figs. 6(a)-6(e)] was dynamically rotated and stretched along one or two coordinates. With our software it is possible to perform any transformation on the matrix, including shifting, stretching, rotating, and $Z$-dimension translation, in real time. This real-time manipulation of the matrix allows for the possibility of an interactive laser tweezer setup in which a parameter such as fluorescence can be detected from a sample and consequently can be quantized by software used to control the matrix by means of a closedloop feedback system. This closed-loop laser tweezer setup can be used for such applications as studying dynamic fluorescence quenching of particles and achieving highly accurate force measurements of biological systems.

We demonstrated another example of real-time, multiple-trap manipulation by drawing characters in a sample by controlling the position of polystyrene beads. The abbreviation ILPB, for our Institute of Lasers, Photonics, and Biophotonics at the University of Buffalo, was dynamically generated in two different manners [Figs. 6(f) and 6(g)]. For Fig. 6(f), 32 beads were trapped by quick $(1.15-\mathrm{ms})$ scanning of the laser beam to a distance of $3.6 \mu \mathrm{m}$ between consecutive sites. In contrast to the stationary structure of Fig. 6(f), that of Fig. 6(g) represents the option when four beads draw the ILPB abbreviation in motion, each bead drawing its own character. We decreased the transition time and the distance between the neighboring sites correspondingly to $460 \mu \mathrm{s}$ and $1.4 \mu \mathrm{m}$, respectively, to achieve this option. Twophoton fluorescence of the moving beads under IR excitation of the trapping beam was detected by an imaging CCD camera with a long $(\sim 2$-s) integration time and was used to image the pattern.

\section{Multitrapping Stiffness Measurement}

The multiple-trap option of laser tweezers has already been found to be useful for patterning the growth of defect structures within colloid crystals..$^{3,4}$ Here an application of this option is demonstrated for calibration of laser tweezer stiffness. The method is based on the measurement of trapping dynamics between two traps and is described in Ref. 15 for twobeam laser tweezers. In our single-beam layout, the stiffness can be estimated in the same way by use of a two-site pattern of the multiple-trap option, with a distance between sites approximately equal to the diameter of the bead $(d)$. We measured the bead position as a function of the time during which the bead escaped from one trap site and was captured by another trap site with the parameters $t_{w}=115 \mathrm{~ms}, t_{t}$ $=1.150 \mathrm{~ms}$, and $d=2.5 \mu \mathrm{m}$. The displacement of the particle during its motion from one trap to another was monitored by a photomultiplier tube signal generated from the backscattering of the aiming $\mathrm{He}-\mathrm{Ne}$ laser beam. The calibration measurements performed previously permit us to monitor the displace-

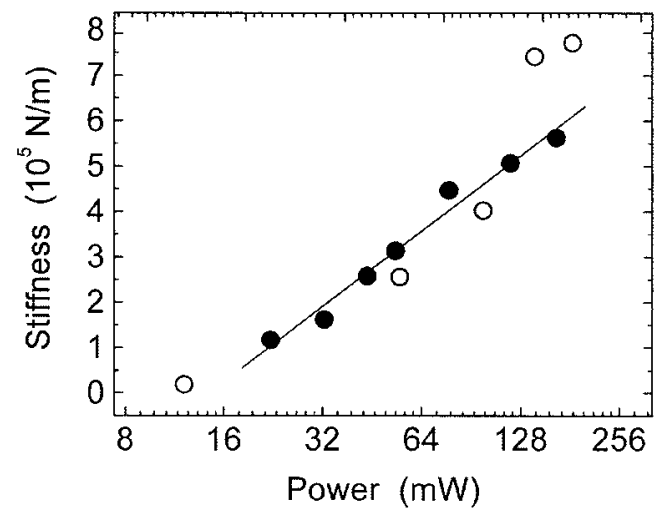

Fig. 7. Power dependence of stiffness measured by use of the multitrap single-beam option and the dual-beam option of optical tweezers. Filled circles, multitrap option of one-beam tweezer; open circles, dual-beam tweezers. 
ment with an accuracy of $\sim 25 \mathrm{~nm}$. The results of the trapping stiffness measurements are presented in Fig. 7 (filled circles) and compared with the results obtained previously for our dual-beam laser tweezer option. ${ }^{14}$ As Fig. 7 depicts, the two results are in good agreement.

\section{Summary}

A multiple-trap approach of quickly scanning a single beam has been characterized and achieved. We have not only demonstrated the existence of an efficient and reliable single-laser-beam multiple-trapping layout for laser tweezers but have also reported the dynamics of the multiple-trap operation. Efficient generation of complex arrays and matrices has been demonstrated in which the number of beads that are capable of being trapped is related to the viscosity of the medium, the refractive index, and the Brownian motion of the sample. We demonstrated an example of the single-laser-beam multiple-trap application by measuring the trap's stiffness in water for our laser tweezer setup. It has also been shown that the trapping efficiency is related to parameters such as illumination time on a bead versus transition time back to the bead and also to the distance by which the return beam deviates from the bead center. Real-time control of the dynamics of the multiple-trap approach is opening new possibilities for measuring forces in biological experiments and possibly bandgap structure formation.

\section{References}

1. P. N. Prasad, Introduction to Biophotonics (Wiley-Interscience, 2003).

2. K. O. Greulich, Micromanipulation by Light in Biology and Medicine (Bizkhauser-Verlag, 1999).

3. J. P. Hoogenboom, D. L. J. Vossen, C. Faivre-Moskalenko, M. Dogterom, and A. van Blaaderenb, "Patterning surfaces with colloidal particles using optical tweezers," Appl. Phys. Lett. 80, 4828-4830 (2002).

4. P. Korda, G. C. Spalding, E. R. Dufresne, and D. G. Grier, "Nanofabrication with holographic optical tweezers," Rev. Sci. Instrum. 73, 1956-1957 (2002).

5. M. P. MacDonald, L. Paterson, W. Sibbett, K. Dholakia, and P. E. Bryant, "Trapping and manipulation of low-index particles in a two-dimensional interferometric optical trap," Opt. Lett. 26, 863-865 (2001).

6. E. R. Dufresne and D. G. Grier, "Optical tweezers arrays and optical substrates created with diffractive optics," Rev. Sci. Instrum. 69, 1975-1977 (1998).

7. A. Jesacher, S. Furhapter, S. Bernet, and M. Ritsch-Marte, "Diffractive optical tweezers in the Fresnel regime," Opt. Express 12, 2243-2250 (2004).

8. R. L. Eriksen, P. C. Mogensen, and J. Gluckstad, "Multiplebeam optical tweezers generated by the generalized phasecontrast method," Opt. Lett. 27, 267-269 (2002).

9. M. Reicherter, T. Haist, E. U. Wagemann, and H. J. Tiziani, "Optical particle trapping with computer-generated holograms written on a liquid crystal display," Opt. Lett. 24, 608-610 (1999).

10. E. R. Dufresne, G. C. Spalding, M. T. Dearing, S. A. Sheets, and D. G. Grier, "Computer-generated holographic optical tweezer arrays," Rev. Sci. Instrum. 72, 1810-1816 (2001).

11. K. Sasaki, M. Koshioka, H. Misawa, N. Kitamura, and H. Masuhara, "Pattern formation and flow control of fine particles by laser-scanning micromanipulation," Opt. Lett. 16, 14631465 (1991).

12. C. Mio, T. Gong, A. Terray, and D. W. M. Marr, "Design of a scanning laser optical trap for multiparticle manipulation," Rev. Sci. Instrum. 71, 2196-2200 (2000).

13. G. J. Brouhard, H. T. Schek, and A. J. Hunt, "Advanced optical tweezers for the study of cellular and molecular biomechanics," IEEE Trans. Biomed. Eng. 50, 121-125 (2003).

14. A. A. Kachynski, A. N. Kuzmin, H. E. Pudavar, D. S. Kaputa, A. N. Cartwright, and P. N. Prasad, "Measurement of optical trapping forces by use of the two-photon-excited fluorescence of microspheres," Opt. Lett. 28, 2288-2290 (2003).

15. W. Singer, S. Bernet, N. Hecker, and M. Ritsch-Marte, "Threedimensional force calibration of optical tweezers," J. Mod. Opt. 47, 2921-2931 (2000). 